\title{
Peces de la fauna de acompañamiento en la pesca industrial de camarón en el Golfo de California, México
}

\author{
Juana López-Martínez ${ }^{1}$, Eloisa Herrera-Valdivia1 ${ }^{1}$ Jesús Rodríguez-Romero ${ }^{2}$ \& Sergio \\ Hernández-Vázquez ${ }^{2}$ \\ 1. Centro de Investigaciones Biológicas del Noroeste, S.C. Km 2.35 Carretera a Las Tinajas, S/N Colonia Tinajas, \\ Guaymas, Sonora, México C. P. 85460; jlopez04@cibnor.mx, eloisa04@cibnor.mx \\ 2. Centro de Investigaciones Biológicas del Noroeste, S.C. Apdo. postal 128 La Paz, B.C.S. C.P. 23000; \\ jrodri04@cibnor.mx, shernan04@cibnor.mx
}

Recibido 19-VII-2009. Corregido 15-III-2010. Aceptado 16-IV-2010.

\begin{abstract}
Bycatch fish species from shrimp industrial fishery in the Gulf of California, Mexico. The shrimp fishery in the Gulf of California is one the most important activities of revenue and employment for communities. Nevertheless, this fishery has also created a large bycatch problem, principally fish. To asses this issue, a group of observers were placed on board the industrial shrimp fleet and evaluated the Eastern side of the Gulf during 2004 and 2005. Studies consisted on $20 \mathrm{~kg}$ samples of the capture for each trawl, and made possible a systematic list of species for this geographic area. Fish represented $70 \%$ of the capture. A total of 51101 fish were collected, belonging to two classes, 20 orders, 65 families, 127 genera, and 241 species. The order Perciformes was the most diverse with 31 families, 78 genera, and 158 species. The best represented families by number of species were: Sciaenidae (34) and Paralichthyidae (18) and Haemulidae and Carangidae (16 each). The best represented genera in number of species were Symphurus (nine) and Diplectrum and Cynoscion (six); other important genera were Larimus and Porichthys with five species each. The best represented species in number were Syacium ovale, Pseudupeneus grandisquamis, Haemulopsis nitidos, Diplectrum pacificum, Synodus scituliceps, Balistes polylepis, Eucinostomus currani, Eucinostomus gracilis, Porichthys analis, Chloroscombrus orqueta, Selene peruviana, Orthopristis reddingi, Etropus crossotus, Scorpaena sonorae and Urobatis halleri. The number of recorded species is notably high, compared with demersal fauna of other areas of the Mexican Pacific, such as Gulf of Tehuantepec (178), Nayarit, Michoacán, Guerrero (174, 120 and 166), Jalisco and Colima (161 species), and those of the Western coast of the Baja California Peninsula (220 species). Rev. Biol. Trop. 58 (3): 925-942. Epub 2010 September 01.
\end{abstract}

Key words: Gulf of California, icthyofauna, shrimp bycatch.

La pesquería industrial de camarón es una de las más importantes en el Golfo de California, debido a que es una fuente significativa de ingresos y empleos para las comunidades a lo largo del Golfo (López-Martínez et al. 2001); a pesar de esto, esta pesquería es también una de las que más contribuye con la problemática de las capturas incidentales en México, generando alrededor de 114 000t de peces, crustáceos y moluscos descartados al año, con una biomasa total estimada en $(90 \pm 45) \times 10^{3} \mathrm{t}$ (Madrid-Vera et al. 2007). Dentro de la fauna de acompañamiento del camarón (FAC), el grupo que mayor abundancia presenta son los peces (Bojorquez 1998, Rábago-Quiroz et al. 2008, RodríguezRomero et al. 2009).

En general, los peces son el grupo más numeroso dentro de los vertebrados, en México se cuenta con una amplia diversidad del grupo que no ha sido evaluada adecuadamente 
(Grainer \& García 1996). Adicionalmente, los inventarios ictiofaunísticos son fundamentales para el conocimiento de la biodiversidad, evaluación de impacto ambiental, estudios biogeográficos y son una herramienta esencial para la administración de las pesquerías con enfoque ecosistémico (Aguilar-Palomino et al. 1996, Rodríguez Romero et al. 2008), ya que provee de criterios en la toma de decisiones para el manejo sustentable de los ecosistemas.

Los listados taxonómicos han retomado importancia en los últimos años, tanto en la península de Baja California (RodríguezRomero et al. 1992, Abitía et al. 1994, De La Cruz-Agüero et al. 1994, Rosales-Casián 1996, Galván-Magaña et al. 2000, Rodríguez Romero et al. 2008) como en el centro y sur del país (Aguilar-Palomino et al. 1996, Madrid-Vera et al. 1998, Moncayo-Estrada et al. 2006), debido a la necesidad de ampliar el conocimiento de nuestros mares, permitiendo con esto sumar esfuerzos para un manejo sustentable de los recursos que en ellos habitan.

Reconociendo la necesidad de ampliar y actualizar el escaso conocimiento sobre la biodiversidad ictiofaunística regional y en particular de las especies de fauna acompañante del camarón (FAC), se presenta un listado sistemático de peces demersales de la plataforma continental de la porción oriental del Golfo de California, región que es considerada como un ecosistema muy dinámico, diverso y con grandes perspectivas de desarrollo.

\section{MATERIALES Y MÉTODOS}

Área de estudio: Durante la temporada de pesca camaronera 2004-2005, que inició en agosto 2004 y concluyó en marzo 2005, se llevó a cabo un programa de "Observadores a bordo" integrado por diez técnicos, cada uno de ellos a bordo de una embarcación camaronera del Estado de Sonora, misma que cubre la plataforma de los estados de Sonora, Sinaloa y Nayarit (Fig. 1). Las embarcaciones camaroneras en Sonora usan redes de arrastre tipo mixto que operan una por banda a ambos lados del barco, el tamaño de malla utilizado en la construcción de las redes camaroneras es de $5.71 \mathrm{~cm}$ en el cuerpo de la red y en el bolso el tamaño mínimo reglamentario de $3.81 \mathrm{~cm}$.

Los muestreos cubrieron estaciones ubicadas a lo largo y ancho de la porción oriental del Golfo de California, entre los 21 13 '37' LN, $105^{\circ} 16^{\prime} 06^{\prime \prime}$ LW y $31^{\circ} 24^{\prime} 35^{\prime \prime}$ LN, $114^{\circ} 22$ '51' LW (Fig. 1), a profundidades variables. Los arrastres tuvieron una duración entre tres y cinco horas a una velocidad aproximada de $5.5 \mathrm{~km} / \mathrm{h}$. Siempre que fue posible una vez efectuado el arrastre, la captura se vació en cubierta y se tomaron aproximadamente $20 \mathrm{~kg}$ de muestra sin seleccionar, dicho tamaño de muestra fue determinado siguiendo criterios estándares (Box et al. 2008). Se procuraron muestras homogéneas y los ejemplares colectados se separaron por familia y género y se almacenaron en la bodega de congelación. La identificación y procesamiento del material biológico se realizó en los laboratorios de pesquerías e ictiología del Centro de Investigaciones Biológicas del Noroeste (CIBNOR, S.C.), sede en Guaymas, Sonora y La Paz, Baja California Sur.

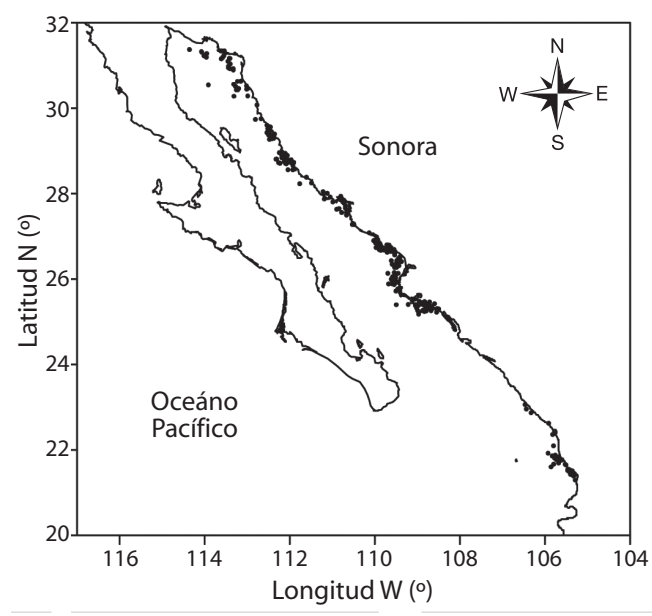

Fig. 1. Estaciones muestreadas a bordo de los barcos camaroneros dentro del Golfo de California, México.

Fig. 1. Sampling stations on board shrimp ships inside the Gulf of California, Mexico. 
A cada ejemplar capturado se le registró la longitud total (LT) y estándar (LS) utilizando un ictiómetro convencional con precisión de $1 \mathrm{~mm}$. La identificación taxonómica de las especies se realizó mediante las claves y descripciones de Jordan \& Evermann (1896-1900), Meek \& Hildebrand (1923-1928), Miller \& Lea (1976), Eschmeyer et al. (1983), Fischer et al. (1995), Robertson \& Allen (2002) y con bibliografía especializada para algunos grupos: Castro-Aguirre \& Espinosa-Pérez (1996) para rayas, Espinosa-Pérez et al. (2004) para tiburones, McPhail (1958) para la familia Sciaenidae, Ginsburg (1958) para Pleuronectiformes, Rosenblatt \& Johnson (1974) para Diplectrum, Walker \& Rosenblatt (1988) para Porichthys, Orr et al. (2000) y Love et al. (2002) para Sebastes. El arreglo sistemático de esta lista taxonómica se realizó siguiendo el criterio de Nelson (2006), los nombres comunes fueron tomados de Love et al. (2002), Robertson \& Allen (2002) y Nelson et al. (2004). Ejemplares de referencia fueron fijados con formaldehído a $10 \%$ y posteriormente preservados en alcohol etílico a $70 \%$. Este material se encuentra en el laboratorio de Investigación Pesquera del Centro de Investigaciones Biológicas del Noroeste, S.C. Unidad Guaymas, para posteriormente ser incorporado a la Colección Ictiológica del Centro. Se estimó la abundancia relativa por especie, considerando el número de cada especie entre el total de individuos muestreados y siguiendo a Rodríguez-Romero et al. (2008), las especies fueron agrupadas en cuatro categorías en función de su abundancia relativa: Especies abundantes (A): aquellas que presentaron una abundancia relativa mayor a $1 \%$; Especies frecuentes $(\mathrm{F})$ : especies con una abundancia relativa entre 0.1 y $0.99 \%$; Especies comunes (C): especies con una abundancia relativa entre 0.01 y $0.099 \%$; Especies raras (R): especies que presentaron una abundancia relativa menor a $0.01 \%$. El análisis de afinidad zoogeográfica fue realizado de acuerdo con el esquema básico de Briggs (1974, 1995) y Walker (1960), con modificaciones de Boschi (2000), Galván et al. (2000), Hastings (2001), Robertson \& Allen (2002), Robertson et al. (2004) y Horn et al. (2006) considerando las siguientes divisiones: PA: Provincia Aleutiana de Isla Nunivak en la Península de Alaska a Puget Sound en Washington, esta es una provincia de aguas templado-frías; PO: Provincia Oregoniana se extiende de Puget Sound a Punta Concepción en California, la porción sur es considerada una zona de transición entre esta provincia y la de San Diego; PS: Provincia de San Diego, de Punta Concepción a Bahía Magdalena en el sur de Baja California, corresponde a aguas templado-cálidas; PC: Provincia de Cortés (sinus californiana) incluyendo la parte sur de Bahía Magdalena y todo el Golfo de California; PM: Provincia Mexicana que incluye la costa de México desde Mazatlán, Sinaloa, hasta el Istmo de Tehuantepec, Oaxaca; PP: Provincia Panámica, se extiende hacia el sur desde El Salvador hasta alrededor de Cabo Blanco, en la parte norte de Perú; POT: Incluye especies de amplia distribución del Pacífico Oriental Tropical, esta región incluye la costa oeste del continente Americano entre $25^{\circ} \mathrm{N}$ en la parte sur de Bahía Magdalena, hasta los $5^{\circ} \mathrm{S}$ en Cabo Blanco parte norte de Perú; CT: Circumtropicales con especies ícticas de amplia distribución en los mares tropicales del mundo.

\section{RESULTADOS}

Se monitorearon 32 viajes de pesca de 13 barcos camaroneros con puerto base en Guaymas, Sonora. La profundidad de muestreo fluctuó de cuatro a $137 \mathrm{~m}$. El número de muestras recolectadas de los 32 viajes fue de 373 , con un peso total de $2331 \mathrm{~kg}$.

Se colectaron 51101 ejemplares, pertenecientes a dos clases, 20 órdenes, 65 familias, 127 géneros y 241 especies (Cuadro 1). El orden Perciformes fue el más diverso con 31 familias, 78 géneros y 158 especies. Las familias mejor representadas en número de especies fueron: Sciaenidae y Paralichthyidae con 34 y 18 especies respectivamente, Haemulidae y Carangidae aportaron 32 especies (16 cada una). Los géneros mejor representados en número de especies fueron Symphurus con nueve y Diplectrum y Cynoscion con 
CUADRO 1

Lista sistemática de peces presentes en la fauna acompañante del camarón en la porción Oriental del Golfo de California, México

TABLE 1

Systematic lists of fish present in the shrimp bycatch in the Oriental side of the Gulf of California, México

\begin{tabular}{|c|c|c|c|c|}
\hline Especie & Nombre común & Nombre inglés & $\begin{array}{l}\text { Abundancia } \\
\text { relativa }\end{array}$ & Afinidad \\
\hline \multicolumn{5}{|l|}{ FILO CHORDATA } \\
\hline \multicolumn{5}{|l|}{ CLASE CHONDRICHTHYES } \\
\hline \multicolumn{5}{|l|}{ SUBCLASE ELASMOBRANCHII } \\
\hline \multicolumn{5}{|l|}{ SUBDIVISION SELACHII } \\
\hline \multicolumn{5}{|l|}{ ORDEN HETERODONTIFORMES } \\
\hline \multicolumn{5}{|l|}{ FAMILIA HETERODONTIDAE } \\
\hline Heterodontus mexicanus Taylor y Castro-Aguirre,1972 & Tiburón perro & Mexican hornshark & $\mathrm{R}$ & POT \\
\hline \multicolumn{5}{|l|}{ ORDEN CARCHARHINIFORMES } \\
\hline \multicolumn{5}{|l|}{ FAMILIA TRIAKIDAE } \\
\hline Mustelus californicus Gill, 1864 & Cazón mamón & Gray smooth hound & $\mathrm{R}$ & POT \\
\hline \multicolumn{5}{|l|}{ ORDEN SQUATINIFORMES } \\
\hline \multicolumn{5}{|l|}{ FAMILIA SQUATINIDAE } \\
\hline Squatina californica Ayres, 1859 & Angelote del Pacífico & Angel shark & $\mathrm{R}$ & PO-PC \\
\hline \multicolumn{5}{|l|}{ SUBDIVISION BATOIDEA } \\
\hline \multicolumn{5}{|l|}{ ORDEN TORPEDINIFORMES } \\
\hline \multicolumn{5}{|l|}{ FAMILIA NARCINIDAE } \\
\hline Diplobatis ommata (Jordan y Gilbert, 1890) & Raya eléctrica diana & Ocellated electric ray & $\mathrm{C}$ & POT \\
\hline Narcine entemedor Jordan y Starks, 1895 & $\begin{array}{l}\text { Raya eléctrica de } \\
\text { Cortés }\end{array}$ & Giante & $\mathrm{R}$ & PC-PP \\
\hline Narcine vermiculatus Breder, 1928 & $\begin{array}{l}\text { Raya eléctrica } \\
\text { vermiculada }\end{array}$ & Vermiculate electric ray & $\mathrm{R}$ & POT \\
\hline \multicolumn{5}{|l|}{ ORDEN RAJIFORMES } \\
\hline \multicolumn{5}{|l|}{ FAMILIA RHINOBATIDAE } \\
\hline Rhinobatos glaucostigma Jordan y Gilbert, 1883 & Guitarra punteada & Splecked guitarfish & F & POT \\
\hline Rhinobatos productus Ayres, 1854 & Guitarra viola & Shovelnose guitarfsh & $\mathrm{F}$ & PO-PM \\
\hline Zapteryx exasperata (Jordan y Gilbert, 1880) & Guitarra rayada & Banded guitarfish & $\mathrm{C}$ & PS-PP \\
\hline \multicolumn{5}{|l|}{ FAMILIA RAJIDAE } \\
\hline Raja equatorialis (Jordan y Bollman, 1890) & Raya ecuatorial & Ecuatorial ray & $\mathrm{R}$ & POT \\
\hline Raja inornata Jordan y Gilbert, 1881 & Raya de California & California ray & $\mathrm{R}$ & PO-PC \\
\hline Raja velezi Chirichigno, 1973 & Raya chilona & Velez ray & $\mathrm{C}$ & POT \\
\hline \multicolumn{5}{|l|}{ ORDEN MYLIOBATIFORMES } \\
\hline \multicolumn{5}{|l|}{ FAMILIA UROLOPHIDAE } \\
\hline Urobatis halleri (Cooper, 1863) & Raya redonda & Haller's round ray & A & POT \\
\hline Urobatis maculatus Garman, 1913 & \multicolumn{2}{|c|}{ Raya redonda de Cortés Spotted round ray } & $\mathrm{F}$ & PS-PC \\
\hline \multicolumn{5}{|l|}{ FAMILIA UROTRYGONIDAE } \\
\hline Urotrygon chilensis (Günther, 1872) & Raya redonda chilena & Chilean round ray & $\mathrm{C}$ & PC-PP \\
\hline Urotryigon rogersi (Jordan y Starks, 1895) & Raya redonda & Roger's round & $\mathrm{C}$ & PC-PP \\
\hline \multicolumn{5}{|l|}{ FAMILIA DASYATIDAE } \\
\hline Dasyatis brevis (Garman, 1880) & Manta raya & Whiptail singray & $\mathrm{C}$ & PO-PP \\
\hline \multicolumn{5}{|l|}{ FAMILIA GYMNURIDAE } \\
\hline Gymnura marmorata (Cooper, 1864) & $\begin{array}{l}\text { Raya mariposa } \\
\text { californiana }\end{array}$ & California butterfly ray & $\mathrm{C}$ & PS-PP \\
\hline \multicolumn{5}{|l|}{ FAMILIA MYLIOBATIDAE } \\
\hline Myliobatis californica Gill, 1865 & Tecolote & Bat eagle ray & $\mathrm{R}$ & PO-PC \\
\hline
\end{tabular}


CUADRO 1 (Continuación)

Lista sistemática de peces presentes en la fauna acompañante del camarón en la porción Oriental

del Golfo de California, México

TABLE 1 (Continued)

Systematic lists of fish present in the shrimp bycatch in the Oriental side of the Gulf of California, México

\begin{tabular}{|c|c|c|c|c|}
\hline Especie & Nombre común & Nombre inglés & $\begin{array}{l}\text { Abundancia } \\
\text { relativa }\end{array}$ & Afinidad \\
\hline \multicolumn{5}{|l|}{ CLASE ACTINOPTERYGII } \\
\hline \multicolumn{5}{|l|}{ SUBCLASE NEOPTERYGII } \\
\hline \multicolumn{5}{|l|}{ DIVISION TELEOSTEI } \\
\hline \multicolumn{5}{|l|}{ ORDEN ALBULIFORMES } \\
\hline \multicolumn{5}{|l|}{ FAMILIA ALBULIDAE } \\
\hline Albula vulpes (Linnaeus, 1758) & Macabí de hebra & Bonefish & $\mathrm{F}$ & POT \\
\hline \multicolumn{5}{|l|}{ ORDEN ANGUILLIFORMES } \\
\hline \multicolumn{5}{|l|}{ SUBORDEN MURAENOIDEI } \\
\hline \multicolumn{5}{|l|}{ FAMILIA MURAENIDAE } \\
\hline Echidna nocturna (Cope, 1872) & Morena pecosa & Freckled moray & $\mathrm{C}$ & PC-PP \\
\hline \multicolumn{5}{|l|}{ SUBORDEN CONGROIDEI } \\
\hline \multicolumn{5}{|l|}{ FAMILIA OPHICHTHIDAE } \\
\hline Ophichthus triserialis (Kaup, 1856) & Tieso del Pacífico & Pacific snake eel & $\mathrm{R}$ & PS-PP \\
\hline $\begin{array}{l}\text { Ophichthus zophochir Jordan y Gilbert, } 1882 \\
\text { FAMILIA MURAENESOCIDAE }\end{array}$ & \multicolumn{2}{|c|}{ FAMILIA MURAENESOCIDAE } & $\mathrm{C}$ & PC-PP \\
\hline \multicolumn{5}{|l|}{ FAMILIA CONGRIDAE } \\
\hline Ariosoma gilberti (Ogilby, 1898) & Anguila jardín & Gilbert's garden eel & $\mathrm{R}$ & PC \\
\hline Bathycongrus macrurus (Gilbert, 1891) & Congrio cabeza corta & Shorthead conger & $\mathrm{C}$ & PC-PP \\
\hline Gnathophis cinctus (Garman, 1899) & Congrio de cola tiesa & Hartail conger & $\mathrm{R}$ & PC-PP \\
\hline \multicolumn{5}{|l|}{ FAMILIA CHLOPSIDAE } \\
\hline \multicolumn{5}{|l|}{ FAMILIA NETTASTOMATIDAE } \\
\hline Hoplunnis pacifica Lane y Stewart, 1968 & Anguila de plata & Silver pike conger & $\mathrm{R}$ & POT \\
\hline \multicolumn{5}{|l|}{ ORDEN CLUPEIFORMES } \\
\hline \multicolumn{5}{|l|}{ SUBORDEN CLUPEOIDEI } \\
\hline \multicolumn{5}{|l|}{ FAMILIA ENGRAULIDAE } \\
\hline Anchovia macrolepidota (Kner, 1863) & Anchoa escamosa & Bigscale anchovy & $\mathrm{C}$ & PC-PM \\
\hline Anchoa nasus (Kner y Steindachner, 1867) & Anchoa trompuda & Longnose anchovy & A & PC-PP \\
\hline Anchoa walkeri Baldwin y Chang, 1970 & Anchoa de Walker & Walker's anchovy & $\mathrm{F}$ & PC-PP \\
\hline Anchoa ischana (Jordan y Gilbert, 1882) & Anchoa chicotera & Siender anchovy & $\mathrm{F}$ & PC-PP \\
\hline Anchoa compressa (Girard, 1858) & Anchoa alta & Deep body anchovy & $\mathrm{C}$ & PS-PM \\
\hline Anchoa lucida (Jordan y Gilbert, 1882) & Anchoa ojitos & Bright anchovy & $\mathrm{R}$ & PC-PP \\
\hline Cetengraulis mysticetus (Günther, 1867) & Anchoveta bocona & Pacific anchoveta & $\mathrm{F}$ & PS-PP \\
\hline \multicolumn{5}{|l|}{ FAMILIA CLUPEIDAE } \\
\hline Harengula thrissina (Jordan y Gilbert, 1882) & Sardina escamuda & Pacific flatiron herring & $\mathrm{R}$ & PC-PP \\
\hline Lile stolifera (Jordan y Gilbert, 1882) & Sardina rayada & Pacific piquitinga & $\mathrm{R}$ & PC-PP \\
\hline Opisthonema libertate (Günther, 1867) & Sardina crinada & Pacific thread herring & $\mathrm{F}$ & PM-PP \\
\hline Sardinops caeruleus (Girard, 1856) & Sardina monterrey & Californian pilchard & $\mathrm{C}$ & POT \\
\hline \multicolumn{5}{|l|}{ FAMILIA PRISTIGASTERIDAE } \\
\hline Opisthopterus dovii (Günther, 1868) & Sardina machete & doven longfin herring & $\mathrm{C}$ & PC-PP \\
\hline Pliosteostoma lutipinnis (Jordan y Gilbert, 1882) & Arenque aleta amarilla & Yellowfin herring & $\mathrm{F}$ & PM-PP \\
\hline
\end{tabular}


CUADRO 1 (Continuación)

Lista sistemática de peces presentes en la fauna acompañante del camarón en la porción Oriental

del Golfo de California, México

TABLE 1 (Continued)

Systematic lists of fish present in the shrimp bycatch in the Oriental side of the Gulf of California, México

\begin{tabular}{|c|c|c|c|c|}
\hline Especie & Nombre común & Nombre inglés & $\begin{array}{l}\text { Abundancia } \\
\text { relativa }\end{array}$ & Afinidad \\
\hline \multicolumn{5}{|l|}{ ORDEN SILURIFORMES } \\
\hline \multicolumn{5}{|l|}{ FAMILIA ARIIDAE } \\
\hline Ariopsis guatemalensis (Günther, 1864) & Bagre cuatete & Blue sea catfish & $\mathrm{C}$ & PC-PP \\
\hline Ariopsis seemanni Günther, 1864 & Bagre tete & Tete sea catfish & $\mathrm{C}$ & PC-PP \\
\hline Ariopsis kessleri (Steindachner, 1877) & Bagre esculpido & $\begin{array}{l}\text { False sculptured sea } \\
\text { catfish }\end{array}$ & $\mathrm{C}$ & PC-PP \\
\hline Ariopsis platypogon (Günther, 1864) & Bagre ojoa & Cominate sea catfish & $\mathrm{F}$ & PC-PP \\
\hline Bagre panamensis (Gill, 1863) & Chihuil & Bagre chihuil & $\mathrm{F}$ & PC-PP \\
\hline Bagre pinnimaculatus (Steindachner, 1877) & Bagre barbón & Long-barbeled sea catfish & $\mathrm{R}$ & PC-PP \\
\hline Cathorops fuerthii (Steindachner, 1877) & Bagre congo & Congo sea catfish & $\mathrm{F}$ & PC-PP \\
\hline \multicolumn{5}{|l|}{ ORDEN ARGENTINIFORMES } \\
\hline \multicolumn{5}{|l|}{ SUBORDEN ARGENTINOIDEI } \\
\hline \multicolumn{5}{|l|}{ FAMILIA ARGENTINIDAE } \\
\hline Argentina sialis Gilbert 1890 & Argentina del pacifico & Pacific argentine & $\mathrm{C}$ & PO-PC \\
\hline \multicolumn{5}{|l|}{ ORDEN AULOPIFORMES } \\
\hline \multicolumn{5}{|l|}{ SUBORDEN SYNODONTOIDEI } \\
\hline \multicolumn{5}{|l|}{ FAMILIA SYNODONTIDAE } \\
\hline Synodus evermanni Jordan y Bollman, 1890 & Chile cadena & Spotted lizardfish & $\mathrm{C}$ & PS-PP \\
\hline Synodus lacertinus Gilbert, 1890 & Chile lagarto & Sauro lizardfish & $\mathrm{C}$ & PS-PP \\
\hline Synodus sechurae Hildebrand, 1946 & Chile iguana & Sechura lizardfish & $\mathrm{C}$ & POT \\
\hline Synodus scituliceps Jordan y Gilbert, 1882 & Chile lagarto & Sortead lizardfish & A & PS-PP \\
\hline \multicolumn{5}{|l|}{ ORDEN OPHIDIIFORMES } \\
\hline \multicolumn{5}{|l|}{ SUBORDEN OPHIDIOIDEI } \\
\hline \multicolumn{5}{|l|}{ FAMILIA OPHIDIIDAE } \\
\hline Lepophidium microlepis (Gilbert, 1890) & Congriperla plateada & Finescale cusk-eel & $\mathrm{R}$ & PS-PP \\
\hline Lepophidium negropinna Hildebrand y Barton, 1949 & Congriperla pinta & Specklefin cusk eel & $\mathrm{R}$ & PS-PP \\
\hline Lepophidium pardale (Gilbert, 1890) & Congriperla leopardo & Leopard cusk eel & $\mathrm{C}$ & POT \\
\hline Lepophidium prorates (Jordan y Bollman, 1890) & Congriperla cornuda & Prowspine cusk eel & $\mathrm{F}$ & POT \\
\hline Ophidion galeoides (Gilbert, 1890) & Congriperla adornada & Spotfin cusk eel & $\mathrm{R}$ & PS-PP \\
\hline \multicolumn{5}{|l|}{ ORDEN BATRACHOIDIFORMES } \\
\hline \multicolumn{5}{|l|}{ FAMILIA BATRACHOIDIDAE } \\
\hline Porichthys analis Hubbs y Schultz, 1939 & Sapo de luto & Darkedge midshipman & A & PC-PM \\
\hline Porichthys margaritatus (Richardson, 1844) & Sapo luminoso & Pearlspot midshipman & $\mathrm{R}$ & PS-PP \\
\hline Porichthys mimeticus (Walker y Rosenblatt, 1988) & Sapo mimético & Mimetic midshipman & $\mathrm{F}$ & $\mathrm{PC}$ \\
\hline Porichthys myriaster Hubbs y Schultz, 1939 & Sapo aleta manchada & Specklefin midshipman & $\mathrm{R}$ & PO-PP \\
\hline Porichthys notatus Girard, 1854 & Sapo aleta lucia & Plainfin midshipman & $\mathrm{R}$ & PO-PS \\
\hline \multicolumn{5}{|l|}{ ORDEN LOPHIIFORMES } \\
\hline \multicolumn{5}{|l|}{ SUBORDEN LOPHIOIDEI } \\
\hline \multicolumn{5}{|l|}{ FAMILIA LOPHIIDAE } \\
\hline Lophiodes caulinaris (Garman, 1899) & Rape rabo manchado & Spottedtail angler & $\mathrm{R}$ & PS-PP \\
\hline Lophiodes spilurus (Garman, 1899) & Rape de hebra & Threadfin angler & $\mathrm{F}$ & PO-PP \\
\hline \multicolumn{5}{|l|}{ SUBORDEN ANTENNARIOIDEI } \\
\hline \multicolumn{5}{|l|}{ FAMILIA ANTENNARIIDAE } \\
\hline Antennarius avalonis Jordan y Starks, 1907 & Ranisapo antenado & Roughbar frogfish & $\mathrm{C}$ & PS-PP \\
\hline
\end{tabular}


CUADRO 1 (Continuación)

Lista sistemática de peces presentes en la fauna acompañante del camarón en la porción Oriental

del Golfo de California, México

TABLE 1 (Continued)

Systematic lists of fish present in the shrimp bycatch in the Oriental side of the Gulf of California, México

\begin{tabular}{|c|c|c|c|c|}
\hline Especie & Nombre común & Nombre inglés & $\begin{array}{l}\text { Abundancia } \\
\text { relativa }\end{array}$ & Afinidad \\
\hline \multicolumn{5}{|l|}{ SUBORDEN OGCOCEPHALIOIDEI } \\
\hline \multicolumn{5}{|l|}{ FAMILIA OGCOCEPHALIDAE } \\
\hline Zalieutes elater (Jordan y Gilbert, 1882) & Murciélago biocelado & Sptted batfish & $\mathrm{R}$ & PO-PP \\
\hline \multicolumn{5}{|l|}{ ORDEN MUGILIFORMES } \\
\hline \multicolumn{5}{|l|}{ FAMILIA MUGILIDAE } \\
\hline Mugil cephalus Linnaeus, 1758 & Lisa cabezona & Flathead grey mullet & $\mathrm{C}$ & PS-PP \\
\hline Mugil curema Valenciennes, 1836 & Lizeta & White mullet & $\mathrm{C}$ & PC-PP \\
\hline \multicolumn{5}{|l|}{ ORDEN GASTEROSTEIFOMES } \\
\hline \multicolumn{5}{|l|}{ SUBORDEN SYNGNATHOIDEI } \\
\hline \multicolumn{5}{|l|}{ FAMILIA SYNGNATHIDAE } \\
\hline Hippocampus ingens Girard, 1858 & Caballito del Pacífico & Pacific seahorse & $\mathrm{R}$ & PO-PP \\
\hline Syngnathus auliscus (Swain, 1882) & Corneticas & Barred pipefish & $\mathrm{R}$ & PO-PP \\
\hline \multicolumn{5}{|l|}{ FAMILIA FISTULARIIDAE } \\
\hline Fistularia commersoni Rüppel, 1835 & Corneta pintada & Bluespotted cornetfish & $\mathrm{R}$ & PC-PP \\
\hline Fistularia corneta Gilbert y Starks, 1904 & Corneta flautera & Pacific cornetfish & $\mathrm{C}$ & PO-PP \\
\hline \multicolumn{5}{|l|}{ ORDEN SCORPAENIFORMES } \\
\hline \multicolumn{5}{|l|}{ SUBORDEN SCORPAENOIDEI } \\
\hline \multicolumn{5}{|l|}{ FAMILIA SCORPAENIDAE } \\
\hline Scorpaena guttata Girard, 1854 & Escorpión californiano & California scorpionfish & $\mathrm{F}$ & PO-PC \\
\hline Scorpaena plumieri mystes Jordan y Starks, 1895 & Escorpión roquero & $\begin{array}{l}\text { Pacific spotted } \\
\text { scorpionfish }\end{array}$ & $\mathrm{F}$ & PO-PP \\
\hline Scorpaena sonorae Jenkins y Evermann, 1889 & Escorpión de Sonora & $\begin{array}{l}\text { Sonora Player } \\
\text { scorpionfish }\end{array}$ & A & PC-PM \\
\hline Scorpaena histrio Jenyns, 1840 & Escorpión jugador & Player scorpionfish & A & PS-PP \\
\hline \multicolumn{5}{|l|}{ SUBORDEN PLATYCEPHALOIDEI } \\
\hline \multicolumn{5}{|l|}{ FAMILIA TRIGLIDAE } \\
\hline Bellator loxias (Jordan, 1897) & Vaca angelita & Barred searobin & A & POT \\
\hline Bellator xenisma (Jordan y Bollman, 1890) & Vaca doble hocico & Splitnose searobin & $\mathrm{F}$ & PS-PP \\
\hline Prionotus albirostris Jordan y Bollman, 1890 & Vaca cariblanca & Whitesnout searobin & $\mathrm{F}$ & POT \\
\hline Prionotus birostratus Richardson, 1844 & Rubio lapón & Two beaken searobin & $\mathrm{C}$ & PC-PP \\
\hline Prionotus ruscarius Gilbert y Starks, 1904 & Vaca rasposa & Common searobin & $\mathrm{F}$ & POT \\
\hline Prionotus stephanophrys Lockington, 1881 & Vaca voladora & Lumptail searobin & $\mathrm{F}$ & PS-PP \\
\hline \multicolumn{5}{|l|}{ ORDEN PERCIFORMES } \\
\hline \multicolumn{5}{|l|}{ SUBORDEN PERCOIDEI } \\
\hline \multicolumn{5}{|l|}{ FAMILIA CENTROPOMIDAE } \\
\hline Centropomus robalito Jordan y Gilbert, 1882 & $\begin{array}{l}\text { Robalo de aleta } \\
\text { amarilla }\end{array}$ & Yellowfin snook & $\mathrm{C}$ & PC-PP \\
\hline \multicolumn{5}{|l|}{ FAMILIA SERRANIDAE } \\
\hline Diplectrum eumelum Rosenblatt y Johnson, 1974 & Serrano guabino & $\begin{array}{l}\text { Orange spotted sand } \\
\text { perch }\end{array}$ & $\mathrm{F}$ & POT \\
\hline Diplectrum macropoma (Günther, 1864) & Serrano mexicano & Mexican sand perch & $\mathrm{R}$ & POT \\
\hline Diplectrum pacificum Meek y Hildebrand, 1925 & Serrano cabaicucho & Inshore sand perch & A & PS-PP \\
\hline Diplectrum maximum Hildebrand, 1946 & Serrano de altura & Torpedo sand perch & $\mathrm{F}$ & PO-PP \\
\hline Diplectrum sciuris Gilbert 1892 & Serrano ardilla & Gulf squirrelfish & $\mathrm{C}$ & PS-PC \\
\hline Diplectrum rostrum Bortone, 1974 & Serrano ardilla & Bridled sand perch & $\mathrm{F}$ & POT \\
\hline Ephinephelus acanthistius (Gilbert, 1892) & Baqueta & Rooster hind & $\mathrm{C}$ & PS-PP \\
\hline Ephinephelus exsul (Fowler, 1944) & Mero diez espinas & Tenspine grouper & $\mathrm{C}$ & PC-PP \\
\hline
\end{tabular}


CUADRO 1 (Continuación)

Lista sistemática de peces presentes en la fauna acompañante del camarón en la porción Oriental

del Golfo de California, México

TABLE 1 (Continued)

Systematic lists of fish present in the shrimp bycatch in the Oriental side of the Gulf of California, México

\begin{tabular}{|c|c|c|c|c|}
\hline Especie & Nombre común & Nombre inglés & $\begin{array}{l}\text { Abundancia } \\
\text { relativa }\end{array}$ & Afinidad \\
\hline Paralabrax auroguttatus Walford, 1936 & Cabrilla extranjera & Goldspotted sand basse & $\mathrm{R}$ & PS-PM \\
\hline Paralabrax maculatofasciatus Steindachner, 1868 & Cabrilla de roca & Spotted sand basse & $\mathrm{F}$ & PS-PM \\
\hline $\begin{array}{l}\text { Rypticus nigripinnis Gill, } 1861 \\
\text { FAMILIA OPISTOGNATHIDAE }\end{array}$ & Jabonero negro & Twice-spotted soapfish & $\mathrm{C}$ & PS-PP \\
\hline $\begin{array}{l}\text { Lonchopisthus sinuscalifornicus Castro-Agirre } \\
\text { y Villavicencio-Garayzar, } 1988\end{array}$ & Bocón cola larga & Longtailed jawfish & $\mathrm{R}$ & $\mathrm{PC}$ \\
\hline $\begin{array}{l}\text { Opistognathus punctatus (Peters, 1869) } \\
\text { FAMILIA PRIACANTHIDAE }\end{array}$ & Microfono & Finespotted jawfish & $\mathrm{R}$ & $\mathrm{PC}$ \\
\hline $\begin{array}{l}\text { Pristigenys serrula (Gilbert, 1891) } \\
\text { FAMILIA MALACANTHIDAE }\end{array}$ & Catalufa semáforo & Popeye catalufa & $\mathrm{C}$ & PO-PP \\
\hline $\begin{array}{l}\text { Caulolatilus affinis Gill, } 1865 \\
\text { FAMILIA CARANGIDAE }\end{array}$ & Pierna & Bighed tilefish & $\mathrm{C}$ & PS-PP \\
\hline Caranx caballus Günther, 1868 & Jurel bonito & Green jack & $\mathrm{C}$ & PS-PP \\
\hline Caranx caninus Günther, 1867 & Jurel toro & Pacific crevalle jak & $\mathrm{R}$ & PS-PP \\
\hline Caranx vinctus Jordan y Gilbert, 1882 & Cocinero & Cocinero & $\mathrm{C}$ & PS-PP \\
\hline Caranx otrynter Jordan y Gilbert, 1883 & Jurel chicuaca registro & Threadfin jack & $\mathrm{R}$ & POT \\
\hline Chloroscombrus orqueta Jordan y Gilbert, 1883 & Horqueta del Pacífico & Pacific bumper & A & PS-PP \\
\hline Hemicaranx leucurus (Günther, 1864) & Jurelito aleta amarilla & Yellowfin jack & $\mathrm{C}$ & POT \\
\hline Hemicaranx zelotes Gilbert, 1898 & Jurel chumbo & Blackin scad & $\mathrm{C}$ & PC-PP \\
\hline Oligoplites altus (Günther, 1868) & Zapatero pelón & Longjaw leatherjack & $\mathrm{C}$ & PS-PP \\
\hline Oligoplites saurus (Bloch y Schneider, 1801) & Zapatero sietecueros & Leatherjack & $\mathrm{F}$ & PS-PP \\
\hline Oligoplites rufulgens (Gilbert y Starks, 1904) & $\begin{array}{l}\text { Zapatero chaqueta de } \\
\text { cuero }\end{array}$ & Shortjaw leatherjack & $\mathrm{F}$ & PM-PP \\
\hline Selar crumenophthalmus (Bloch, 1793) & Chicharro ojón & Bigeye scad & $\mathrm{C}$ & PC-PP \\
\hline Selene brevoorti (Gill, 1863) & Jorobado mexicano & Mexican lookdown & $\mathrm{R}$ & PC-PP \\
\hline Selene oerstedii Lütken, 1880 & Jorobado chapeta & Mexican moontfish & $\mathrm{C}$ & PC-PP \\
\hline Selene peruviana (Guichenot, 1866) & Jorobado papelillo & Pacific moontfish & A & PS-PP \\
\hline Trachinotus kennedyi Steindachner, 1876 & Pámpano plateado & Blackblotch pompano & $\mathrm{R}$ & PS-PP \\
\hline $\begin{array}{l}\text { Trachinotus paitensis Cuvier, } 1832 \\
\text { FAMILIA NEMATISTIIDAE }\end{array}$ & Pámpano paloma & Paloma pompano & $\mathrm{C}$ & PS-PP \\
\hline $\begin{array}{l}\text { Nematistius pectorales (Gill, 1862) } \\
\text { FAMILIA LUTJANIDAE }\end{array}$ & Peje gallo & Roosterfish & $\mathrm{R}$ & PS-PP \\
\hline Lutjanus argentiventris (Peters, 1869) & Pargo amarillo & Yellow snapper & $\mathrm{C}$ & PS-PP \\
\hline Lutjanus guttatus (Steindachner, 1869) & Pargo lunarejo & Spotted rose snapper & $\mathrm{F}$ & PC-PP \\
\hline Lutjanus jordani (Gilbert, 1897) & Pargo rubirrubia & Jordan's snapper & $\mathrm{R}$ & PC-PP \\
\hline FAMILIA GERREIDAE & & & & \\
\hline Diapterus aureolus (Jordan y Gilbert, 1882) & Mojarra palometa & Golden mojarra & A & PC-PP \\
\hline Diapterus peruvianus (Cuvier, 1830) & $\begin{array}{l}\text { Mojarra de aleta } \\
\text { amarilla }\end{array}$ & Peruvian mojarra & A & PC-PP \\
\hline Eucinostomus currani (Zahuranec, 1980) & Mojarra tricolor & Pacific flagfin majorra & $\mathrm{A}$ & PC-PP \\
\hline Eucinostomus dowii (Gill, 1863) & Mojarra manchita & Silver mojarra & A & PC-PP \\
\hline Eucinostomus entomelas Zahuranec, 1980 & Mojarra mancha negra & Dark spot majorra & $\mathrm{A}$ & PC-PP \\
\hline Eucinostomus gracilis (Gill, 1862) & Mojarra charrita & Graceful mojarra & A & PC-PP \\
\hline Eugerres axillaris (Günther, 1864) & Mojarra malacapa & Black axillary mojarra & $\mathrm{F}$ & PC-PP \\
\hline Eugerres lineatus (Humboldt, 1821) & Mojarra china & Streaked mojarra & $\mathrm{C}$ & PC-PP \\
\hline
\end{tabular}


CUADRO 1 (Continuación)

Lista sistemática de peces presentes en la fauna acompañante del camarón en la porción Oriental

del Golfo de California, México

TABLE 1 (Continued)

Systematic lists of fish present in the shrimp bycatch in the Oriental side of the Gulf of California, México

\begin{tabular}{|c|c|c|c|c|}
\hline Especie & Nombre común & Nombre inglés & $\begin{array}{l}\text { Abundancia } \\
\text { relativa }\end{array}$ & Afinidad \\
\hline \multicolumn{5}{|l|}{ FAMILIA HAEMULIDAE } \\
\hline Conodon serrifer Jordan y Gilbert, 1882 & Ronco ofensivo & Armed grunt & $\mathrm{C}$ & PC-PP \\
\hline Haemulon flaviguttatum Gill, 1862 & Burro de cortèz & Yellowspotted grunt & $\mathrm{R}$ & PC-PP \\
\hline Haemulon maculicauda (Gill, 1862) & $\begin{array}{l}\text { Ronco de rabo } \\
\text { manchado }\end{array}$ & Spottail grunt & $\mathrm{C}$ & $\begin{array}{l}\text { PS-PP } \\
\text { PS-PP }\end{array}$ \\
\hline Haemulon steindachneri (Jordan y Gilbert, 1882) & Burro latino & Latin grunt & $\mathrm{F}$ & POT \\
\hline Haemulopsis axillaris (Steindachner, 1869) & Ronco catalineta & Yellowstripe grunt & A & PC-PP \\
\hline Haemulopsis leuciscus (Günther, 1864) & Roncacho & White grunt & $\mathrm{F}$ & PS-PP \\
\hline Haemulopsis elongatus (Steindachner, 1879) & Ronco alargado & Elongate grunt & $\mathrm{F}$ & PM-PP \\
\hline Haemulopsis nitidus (Steindachner, 1869) & Ronco brillante & Shinning grunt & A & PC-PP \\
\hline Orthopristis cantharinus (Jenyns, 1840) & Burrito brin & Sheephead grunt & $\mathrm{F}$ & PC \\
\hline Orthopristis reddingi Jordan y Richardson, 1985 & Burrito roncacho & Bronze striped grunt & A & PM \\
\hline Orthopristis chalceus (Günther, 1864) & Burrito corvovado & Brassy grunt & $\mathrm{F}$ & POT \\
\hline Pomadasys panamensis (Steindachner, 1876) & Ronco mapache & Panama grunt & A & PC-PP \\
\hline Pomadasys bayanus Jordan y Evermann, 1898 & Ronco blanquillo & Purplemouth grunt & $\mathrm{C}$ & PC-PP \\
\hline Pomadasys macracanthus (Günther, 1864) & Ronco bacoco & Longspine grunt & $\mathrm{C}$ & PC-PP \\
\hline Xenistius californiensis (Steindachner, 1876) & Pajarillo & Salema & $\mathrm{F}$ & PS-PP \\
\hline Xenichthys xanti Gill, 1863 & Roncador ojón & Longfin salema & $\mathrm{F}$ & PS-PP \\
\hline \multicolumn{5}{|l|}{ FAMILIA POLYNEMIDAE } \\
\hline Polydactylus approximans (Lay y Bennett, 1839) & Barbudo azul & Blue bobo & $\mathrm{F}$ & PS-PP \\
\hline Polydactylus opercularis Gill, 1863 & Barbudo amarillo & Yellow bobo & $\mathrm{F}$ & PS-PP \\
\hline \multicolumn{5}{|l|}{ FAMILIA SCIAENIDAE } \\
\hline Bairdiella armata Gill, 1863 & Corvina armada & Armed croaker & $\mathrm{C}$ & PC-PP \\
\hline Bairdiella incistia (Jordan y Gilbert, 1882) & Ronco roncacho & Ronco croaker & $\mathrm{R}$ & PC-PP \\
\hline Cheilotrema saturnum (Girard, 1858) & Corvina roncacho & Black croakrer & $\mathrm{R}$ & PO-PC \\
\hline Cynoscion othonopterus Jordan y Gilbert, 1882 & Corvina golfina & Guf weakfish & $\mathrm{R}$ & PC-PP \\
\hline $\begin{array}{l}\text { Cynoscion nannus Castro-Aguirre y Arvizu-Martínez, } \\
1976\end{array}$ & $\begin{array}{l}\text { Corvina enana de } \\
\text { altamar }\end{array}$ & Dwarf weakfish & $\mathrm{C}$ & PC-PP \\
\hline Cynoscion reticulatus (Günther, 1864) & Corvina rallada & Striped weakfish & $\mathrm{F}$ & PC-PP \\
\hline Cynoscion squamipinnis (Günther, 1867) & Corvina aguada & Weakfish & $\mathrm{C}$ & PC-PP \\
\hline Cynoscion stolzmanni (Steindachner, 1879) & Corvina pelona & Stolzmann's weakfish & $\mathrm{F}$ & PC-PP \\
\hline Cynoscion xanthulus Jordan y Gilbert, 1881 & $\begin{array}{l}\text { Corvina de boca } \\
\text { amarilla }\end{array}$ & Orangemouth weakfish & $\mathrm{C}$ & PS-PP \\
\hline Isopisthus remifer Jordan y Gilbert, 1882 & Corvineta azul & Silver wakfish & $\mathrm{C}$ & PS-PP \\
\hline Larimus argenteus (Gill, 1863) & Corvineta boquinete & Silver drum & $\mathrm{C}$ & PC-PP \\
\hline Larimus effulgens Gilbert en Jordan y Evermann, 1898 & Corvineta chata & Shinning drum & $\mathrm{F}$ & PC-PP \\
\hline Larimus pacificus Jordan y Bollman, 1890 & Corvineta tambor & Pacific drum & $\mathrm{C}$ & POT \\
\hline Larimus acclivis Jordan y Brisol, 1898 & $\begin{array}{l}\text { Corvineta de estrías } \\
\text { amarillas }\end{array}$ & Steeplined drum & $\mathrm{F}$ & PC-PP \\
\hline Menticirrhus elongatus (Günther, 1864) & Berrugata bocadulce & Pacific kingcroaker & $\mathrm{R}$ & PC-PP \\
\hline Menticirrhus panamensis (Steindachner, 1875) & Berrugata gurrubata & Panama kingcroaker & $\mathrm{C}$ & PC-PP \\
\hline Menticirrhus nasus (Günther, 1868) & Berrugata real & Highfin king croaker & $\mathrm{C}$ & PC-PP \\
\hline Micropogonias altipinnis (Günther, 1864) & Corvina berrugata & Tallfin croaker & $\mathrm{F}$ & PC-PP \\
\hline Micropogonias ectenes (Jordan y Gilbert, 1881) & Corvina bocadulce & Siender croaker & $\mathrm{C}$ & PS-PM \\
\hline Micropogonias megalops (Gilbert, 1890) & Corvina ojo grande & Bigeye croaker & A & PC-PM \\
\hline Ophioscion scierus (Jordan y Gilbert, 1884) & Corvineta zorra & Point tuza croaker & $\mathrm{C}$ & POT \\
\hline
\end{tabular}


CUADRO 1 (Continuación)

Lista sistemática de peces presentes en la fauna acompañante del camarón en la porción Oriental del Golfo de California, México

TABLE 1 (Continued)

Systematic lists of fish present in the shrimp bycatch in the Oriental side of the Gulf of California, México

\begin{tabular}{|c|c|c|c|c|}
\hline Especie & Nombre común & Nombre inglés & $\begin{array}{l}\text { Abundancia } \\
\text { relativa }\end{array}$ & Afinidad \\
\hline Odontoscion xanthops Gill, 1898 & $\begin{array}{l}\text { Corvinata de ojo } \\
\text { amarillo }\end{array}$ & Yelloweye croaker & $\mathrm{C}$ & \\
\hline Paralonchurus goodei Gilbert, 1898 & Corvina mimis & Goode croaker & $\mathrm{R}$ & PC-PP \\
\hline Paralonchurus rathbuni Jordan y Bollman, 1890 & Corvina coco & Bearded banded croaker & $\mathrm{R}$ & PM-PP \\
\hline Pareques viola (Gilbert, 1898) & Corvinita camiseta & Odd highhat & $\mathrm{R}$ & PC-PP \\
\hline Roncador stearnsii (Steindachner, 1876) & $\begin{array}{l}\text { Corvina de aleta } \\
\text { manchada }\end{array}$ & Spotfin croaker & $\mathrm{C}$ & PO-PC \\
\hline Totoaba macdonaldi (Gilbert, 1890) & Totoaba & Totoaba & $\mathrm{R}$ & END \\
\hline Stellifer furthii Steindachner, 1876 & Corvina blanca & White stardrum & $\mathrm{F}$ & PP \\
\hline Stellifer ericymba (Jordan y Gilbert, 1882) & Corvinilla mayita & Yawning stardrum & $\mathrm{C}$ & PM-PS \\
\hline Umbrina analis Günther, 1868 & Berrugato espinoso & Longspine drum & $\mathrm{R}$ & PC-PP \\
\hline Umbrina roncador Jordan y Gilbert, 1882 & $\begin{array}{l}\text { Verrugato de aleta } \\
\text { amarilla }\end{array}$ & Yellowfin drum & $\mathrm{C}$ & PS-PP \\
\hline Umbrina wintersteeni Walker y Radford, 1992 & Verrugato & Wintersteen drum & $\mathrm{R}$ & PS-PP \\
\hline Umbrina xanti Gill, 1862) & Verrugato roncador & Polla drum & $\mathrm{F}$ & PM-PP \\
\hline FAMILIA MULLIDAE & & & & \\
\hline Mulloidichthys dentatus Gill, 1862 & Chivato & Mexican goatfish & $\mathrm{F}$ & PS-PP \\
\hline $\begin{array}{l}\text { Pseudupeneus grandisquamis (Gill, 1863) } \\
\text { FAMILIA CHAETODONTIDAE }\end{array}$ & Chivo escamudo & Bigscale goatfish & A & PS-PP \\
\hline Chaetodon humeralis Günther, 1860 & Mariposa muñeca & Threebanded butterflyfish & $\mathrm{C}$ & PS-PP \\
\hline $\begin{array}{l}\text { SUBORDEN LABROIDEI } \\
\text { FAMILIA LABRIDAE }\end{array}$ & & & & \\
\hline Halichoeres chierchiae di Caporiacco, 1948 & Señorita herida & Wounded wrasse & $\mathrm{R}$ & PC-PP \\
\hline Halichoeres semicinctus (Ayres, 1859) & Señorita piedrera & Rock wrasse & $\mathrm{R}$ & PS-PM \\
\hline $\begin{array}{l}\text { Polylepion cruentum Gomon, } 1977 \\
\text { SUBORDEN TRACHINOIDEI } \\
\text { FAMILIA URANOSCOPIDAE }\end{array}$ & Vieja sangradora & Bledding wrasse & $\mathrm{R}$ & PM-PP \\
\hline Astroscopus zephyreus Gilbert y Starks, 1897 & Murciélago perro & Pacific stargazer & $\mathrm{R}$ & PO-PP \\
\hline $\begin{array}{l}\text { Kathetostoma averruncus Jordan y Bollmann, } 1890 \\
\text { FAMILIA EPHIPPIDAE }\end{array}$ & Miracielo sapo & Smooth stargazer & $\mathrm{R}$ & PO-PP \\
\hline $\begin{array}{l}\text { Chaetodipterus zonatus (Girard, 1858) } \\
\text { SUBORDEN GOBIOIDEI } \\
\text { FAMILIA GOBIIDAE }\end{array}$ & Peluquero & Pacific spadefish & $\mathrm{C}$ & PC-PP \\
\hline Microgobius erectus Ginsburg, 1938 & Gobio chato & Erect goby & $\mathrm{C}$ & PC \\
\hline $\begin{array}{l}\text { Microgobius brevispinnis Ginsburg, } 1939 \\
\text { SUBORDEN SCOMBROIDEI } \\
\text { FAMILIA TRICHIURIDAE }\end{array}$ & Gobio de Balboa & Balboa goby & $\mathrm{C}$ & PC-PP \\
\hline $\begin{array}{l}\text { Trichiurus lepturus (Linnaeus, 1758) } \\
\text { FAMILIA SCOMBRIDAE }\end{array}$ & Pez sable & Largedhead hairtail & $\mathrm{C}$ & PS-PP \\
\hline Scomber japonicus Houttuyn, 1782 & Macarela estornino & Chub mackerel & $\mathrm{C}$ & $\mathrm{T}$ \\
\hline $\begin{array}{l}\text { Scomberomorus sierra Jordan y Starks } 1895 \\
\text { FAMILIA SPHYRAENIDAE }\end{array}$ & Sierra común & Pacific sierra & $\mathrm{C}$ & PS-PP \\
\hline Sphyraena ensis Jordan y Gilbert, 1882 & Barracuda & Mexican barracuda & $\mathrm{C}$ & PC-PP \\
\hline
\end{tabular}


CUADRO 1 (Continuación)

Lista sistemática de peces presentes en la fauna acompañante del camarón en la porción Oriental

del Golfo de California, México

TABLE 1 (Continued)

Systematic lists of fish present in the shrimp bycatch in the Oriental side of the Gulf of California, México

\begin{tabular}{|c|c|c|c|c|}
\hline Especie & Nombre común & Nombre inglés & $\begin{array}{l}\text { Abundancia } \\
\text { relativa }\end{array}$ & Afinidad \\
\hline \multicolumn{5}{|l|}{ SUBORDEN STROMATEOIDEI } \\
\hline \multicolumn{5}{|l|}{ FAMILIA STROMATEIDAE } \\
\hline Peprilus medius (Peters, 1869) & Palometa & Pacific harvestfish & $\mathrm{F}$ & POT \\
\hline Peprilus snyderi Gilbert y Starks, 1904 & Palometa salema & Salema butterfish & $\mathrm{C}$ & PS-PP \\
\hline \multicolumn{5}{|l|}{ ORDEN PLEURONECTIFORMES } \\
\hline \multicolumn{5}{|l|}{ SUBORDEN PLEURONECTOIDEI } \\
\hline \multicolumn{5}{|l|}{ FAMILIA PARALICHTHYIDAE } \\
\hline Ancylopsetta dendritica Gilbert, 1890 & Lenguado tres ojos & Three spot flounder & $\mathrm{R}$ & PM-PP \\
\hline Citharichthys fragilis Gilbert, 1890 & Lenguado flaco & Gulf sanddab & $\mathrm{C}$ & PO-PC \\
\hline Citharichthys gilberti Jenkins y Evermann, 1889 & Lenguado tapadero & bigmouthssanddab & $\mathrm{C}$ & PM-PP \\
\hline Citharichthys gordae Beebe y Tee-Van, 1938) & Lenguado escondido & Mimic sanddab & $\mathrm{C}$ & PC \\
\hline Citharichthys xanthostigma Gilbert, 1890 & Lenguado alón & Longfin sanddab & $\mathrm{R}$ & PO-PP \\
\hline Cyclopsetta panamensis (Steindachner, 1876) & Lenguado panámico & God's flounder & $\mathrm{C}$ & PC-PP \\
\hline Cyclopsetta querna Jordan y Bollman, 1890 & Lenguado dientón & Toothed flounder & $\mathrm{C}$ & PC-PP \\
\hline Etropus crossotus Jordan y Gilbert, 1882 & Lenguado ribete & Fringed flounder & A & PS-PP \\
\hline Etropus peruvianus Hildebrand, 1946 & Lenguado zapatilla & Peruvian flounder & A & PC-PP \\
\hline Hippoglossina bollmani Gilbert, 1890 & Lenguado pintado & Spotted flounder & $\mathrm{R}$ & PS-PP \\
\hline Hippoglossina stomata Eigenmann y Eigenmann, 1890 & Lenguado bocón & Bigmouth flounder & $\mathrm{R}$ & PC-PM \\
\hline Hippoglossina tetrophthalma (Gilbert, 1890) & Lenguado cuatrojos & Fourspot flounder & $\mathrm{C}$ & PS-PP \\
\hline Paralichthys aestuarius Gilbert y Scofield, 1898 & Lenguado de Cortés & Cortez flounder & $\mathrm{C}$ & PS-PC \\
\hline Paralichthys californicus (Ayres, 1859) & Lenguado californiano & California flounder & $\mathrm{R}$ & PO-PC \\
\hline Paralichthys woolmani Jordan y Williams, 1897 & Lenguado huarache & Speckled flounder & $\mathrm{F}$ & PS-PP \\
\hline Syacium latifrons (Jordan y Gilbert, 1882) & Lenguado playero & Beach flounder & $\mathrm{F}$ & POT \\
\hline Syacium ovale (Günther, 1864) & Lenguado ovalado & Oval flounder & A & POT \\
\hline Xystreurys liolepis Jordan y Gilbert, 1880 & $\begin{array}{l}\text { Lenguado cola de } \\
\text { abanico }\end{array}$ & Fantail flounder & $\mathrm{C}$ & PO-PC \\
\hline \multicolumn{5}{|l|}{ FAMILIA PLEURONECTIDAE } \\
\hline Hypsopsetta guttulata (Girard, 1856) & Platija diamante & Diamond turbot & $\mathrm{R}$ & PO-PC \\
\hline Pleuronichthys ocellatus Starks y Thompson, 1910 & Platija ocelada & Ocellated turbot & $\mathrm{F}$ & PC \\
\hline Pleuronichthys ritteri Starks y Morris, 1907 & Platija moteada & Spotted turbot & $\mathrm{C}$ & PO-PS \\
\hline $\begin{array}{l}\text { Pleuronichthys verticalis Jordan y Gilbert, } 1880 \\
\text { FAMILIA BOTHIDAE }\end{array}$ & Platija cornuda & Homyhead turbot & $\mathrm{F}$ & PO-PC \\
\hline Bothus leopardinus (Günther, 1862) & $\begin{array}{l}\text { Lenguado leopardo del } \\
\text { Pacífico }\end{array}$ & Pacific leopard flounder & A & POT \\
\hline Perissias taeniopterus (Gilbert, 1890) & Lenguado bandera & Stripped fin flounder & $\mathrm{R}$ & PS-PP \\
\hline \multicolumn{5}{|l|}{ FAMILIA CYNOGLOSSIDAE } \\
\hline Symphurus atramentatus Jordan y Bollman, 1890 & Lengua de vaca & Inskpot tongefish & $\mathrm{C}$ & PC-PP \\
\hline Symphurus atricaudus (Jordan y Gilbert, 1880) & Lengua californiana & California tongefish & $\mathrm{R}$ & PO-PP \\
\hline Symphurus elongatus (Günther, 1868) & Lengua esbelta & Elongate tongefish & $\mathrm{C}$ & PM-PP \\
\hline Symphurus fasciolaris Gilbert, 1892 & Lengua listada & Banded tonge fish & $\mathrm{C}$ & \\
\hline Symphurus chabanaudi Mahadeva y Munroe, 1990 & Lengua cachete prieto & Chabanaud's tongefish & $\mathrm{F}$ & PC-PP \\
\hline Symphurus leei Jordan y Bollman, 1889 & Lengua colinegra & Lee's tonguefish & $\mathrm{R}$ & PC-PP \\
\hline Symphurus melanurus Clark, 1936 & Lengua lucia & Drab tonguefish & $\mathrm{C}$ & POT \\
\hline Symphurus williamsi Jordan y Culver, 1895 & Lengua de Williams & William's tongefish & $\mathrm{C}$ & PC-PP \\
\hline Symphurus callopterus Munroe y Mahadeva, 1989 & Lengua chocolate & Chocolate tongefish & $\mathrm{R}$ & PC-PP \\
\hline
\end{tabular}


CUADRO 1 (Continuación)

Lista sistemática de peces presentes en la fauna acompañante del camarón en la porción Oriental del Golfo de California, México

TABLE 1 (Continued)

Systematic lists of fish present in the shrimp bycatch in the Oriental side of the Gulf of California, México

\begin{tabular}{|c|c|c|c|c|}
\hline Especie & Nombre común & Nombre inglés & $\begin{array}{l}\text { Abundancia } \\
\text { relativa }\end{array}$ & Afinidad \\
\hline \multicolumn{5}{|l|}{ FAMILIA ACHIRIDAE } \\
\hline Achirus mazatlanus (Steindachner, 1869) & Pez sol & Mazatlan sole & A & PS-PP \\
\hline Trinectes fonsecensis (Günther, 1862) & Sol rayado & Spottedfin sole & $\mathrm{R}$ & PS-PP \\
\hline \multicolumn{5}{|l|}{ ORDEN TETRAODONTIFORMES } \\
\hline \multicolumn{5}{|l|}{ SUBORDEN BALISTOIDEI } \\
\hline \multicolumn{5}{|l|}{ FAMILIA BALISTIDAE } \\
\hline Balistes polylepis (Steindachner, 1876 ) & Cochi ribereño & Finescale triggerfish & A & PO-PP \\
\hline \multicolumn{5}{|l|}{ FAMILIA MONACANTHIDAE } \\
\hline Aluterus monoceros Linnaeus, 1758 & Puerco unicornio & Unicorn leatherjacket & $\mathrm{R}$ & $\mathrm{CT}$ \\
\hline \multicolumn{5}{|l|}{ SUBORDEN TETRAODONTOIDEI } \\
\hline \multicolumn{5}{|l|}{ FAMILIA TETRAODONTIDAE } \\
\hline Lagocephalus lagocephalus (Linnaeus, 1758) & Botete oceánico & Ocanic puffer & $\mathrm{F}$ & CT \\
\hline Sphoeroides annulatus (Jenyns, 1843) & Botete diana & Bullseye puffer & A & PO-PP \\
\hline Sphoeroides lispus Walker, 1996 & Botete liso & Smooth puffer & $\mathrm{F}$ & PS-PC \\
\hline Sphoeroides lobatus (Steindachner, 1870) & Botete verrugoso & Longnose puffer & $\mathrm{F}$ & PS-PP \\
\hline Sphoeroides sechurae Hildebrand, 1946 & Botete peruano & Peruvian puffer & $\mathrm{F}$ & POT \\
\hline \multicolumn{5}{|l|}{ FAMILIA DIODONTIDAE } \\
\hline Diodon holocanthus Linnaeus, 1758 & Pez erizo mapache & Balloonfish & $\mathrm{R}$ & $\mathrm{CT}$ \\
\hline Diodon hystrix Linnaeus, 1758 & Pez erizo espinoso & $\begin{array}{l}\text { Black spotted } \\
\text { porcupinefsh }\end{array}$ & $\mathrm{R}$ & $\mathrm{T}$ \\
\hline
\end{tabular}

Especie abundante (A), especie frecuente $(\mathrm{F})$, especie común $(\mathrm{C})$ y especie rara $(\mathrm{R})$. $\mathrm{PO}=$ Provincia oregoniana; $\mathrm{PS}=\mathrm{Provincia}$ de San Diego; PA=Provincia aleutiana; $\mathrm{CT}=$ Especie circuntropical; POT=Pacífico oriental tropical; $\mathrm{PC}=$ Provincia de Cortés; $\mathrm{PM}=$ Provincia mexicana; $\mathrm{PP}=$ Provincia panámica; $\mathrm{T}=$ Especie transpacífica; $\mathrm{END}=$ Especie endémica del Golfo de California.

seis especies, otros géneros importantes fueron Larimus y Porichthys, con cinco especies cada uno. El intervalo de tallas de todos los peces varió de 2.2 a $94.7 \mathrm{~cm}$ de longitud total. La especie Pseudupeneus grandisquamis (Gill 1863) registró la menor talla $(2.2 \mathrm{~cm}$ LT) y Fistularia corneta (Gilbert \& Starks, 1904) la mayor ( $94.7 \mathrm{~cm} \mathrm{LT).} \mathrm{Las} \mathrm{especies} \mathrm{más}$ importantes por su abundancia relativa fueron el lenguado ovalado Syacium ovale (Günther, 1864), el chivo escamudo Pseudupeneus grandisquamis (Gill, 1863), el ronco brillante Haemulopsis nitidos (Steindachner, 1869), el serrano cabaicucho Diplectrum pacificum Meek \& Hildebrand, 1925, el lagarto camote
Synodus scituliceps Jordan y Gilbert, 1882, el cochi ribereño Balistes polylepis Steindachner, 1876, la mojarra tricolor Eucinostomus currani Zahuranec, 1980, la charrita Eucinostomus gracilis (Gill, 1862), el sapo de luto Porichthys analis Hubbs \& Schultz, 1939, la horqueta del Pacífico Chloroscombrus orqueta Jordan \& Gilbert, 1883, el jorobado mexicano Selene peruviana (Guichenot, 1866), el burrito roncacho Orthopristis reddingi Jordan \& Richardson, 1985, el lenguado ribete Etropus crossotus Jordan \& Gilbert, 1882, el escorpión de Sonora Scorpaena sonorae Jenkins \& Evermann, 1889 y la raya redonda de aguijón Urobatis halleri (Cooper, 1863). 
Este estudio presenta nuevos registros o ampliación de rango de distribución en la zona para el congrio de cola tiesa Gnathophis cinctus (Garman, 1899), el bagre congo Cathorops fuerthii (Steindachner, 1877), el jurel de hebra Caranx otrynter Jordan \& Gilbert, 1883, el Jorobado chapeta Selene oerstedii Lütken 1880, el pargo rubirrubia Lutjanus jordani (Gilbert, 1897), el coco Paralonchurus rathbuni (Jordan \& Bollman 1890), la corvinilla mayita Stellifer oscitans (Jordan \& Gilbert, 1882) y la corvinita listada Pareques viola (Gilbert, 1898). Acontecimientos similares de un incremento de nuevos registro o rango de distribución de especies de peces demersales en los últimos años también han sido reportados por Rodríguez-Romero et al. (2008) en la costa occidental de Baja California.

La distribución biogeográfica estuvo dominada por especies encontradas en las provincias de cortes-panámica, el traslape de la provincia sandieguina-panámica y por especies del Pacifico Oriental Tropical (Fig. 2).

\section{DISCUSIÓN}

La riqueza de especies de peces demersales registrada en este estudio (241 especies) para el Golfo de California es significativamente alta en comparación con otros estudios realizados, pero puede aumentar si se considera el aporte de especies de las zonas costeras y pelágicas. Los trabajos realizados previamente para esta región con relación al conocimiento de la biodiversidad de peces han sido escasos, puntuales y dispersos en espacio y tiempo (Walker 1960, Castro-Aguirre et al. 1970, Chávez \& Arvizu 1972, Thompson et al. 1979, Pérez-Mellado 1980, Pérez-Mellado et al. 1982, Van der Heiden \& Findley 1988, Nava Romo 1995). Este

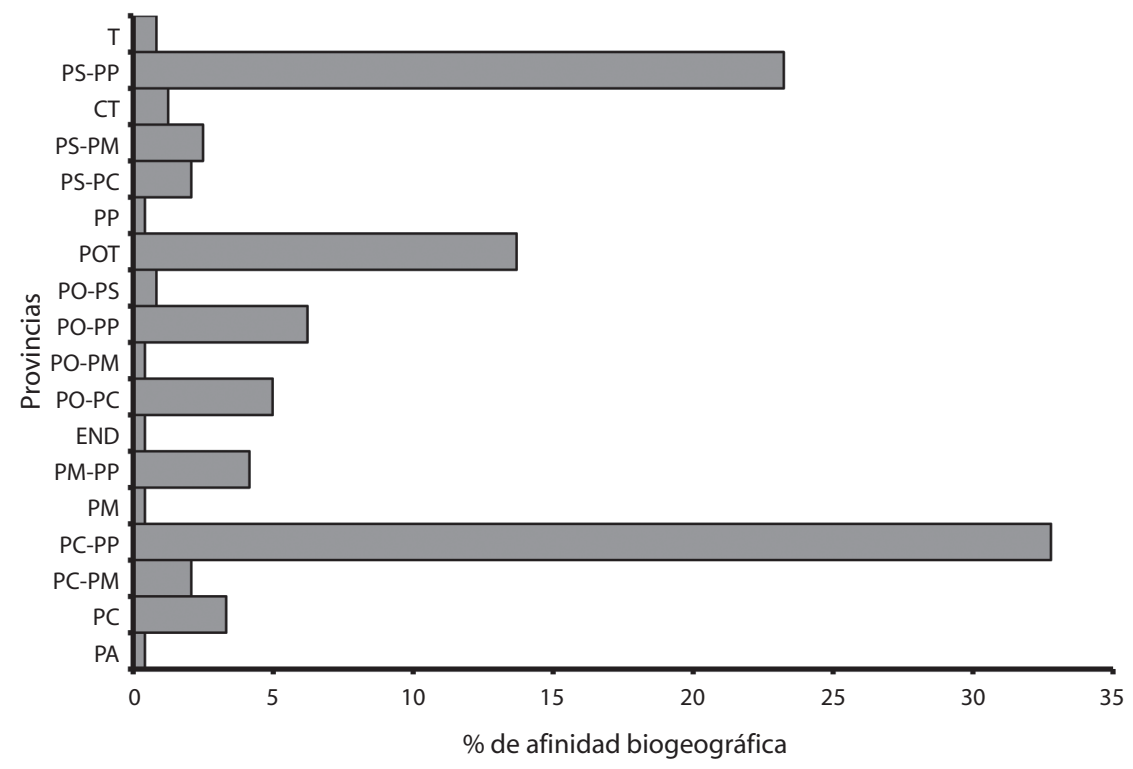

Fig. 2. Porcentaje de afinidad biogeográfica de la fauna de peces del Golfo de California. (PA: Provincia aleutiana, PO: Provincia oregoniana, PS: Provincia de San Diego, PC: Provincia de Cortés, PM: Provincia mexicana, PP: Provincia panámica, POT: Pacífico oriental tropical, CT: Especies circumtropicales, T: Especies transpacíficas, END: Especies endémicas).

Fig. 2. Percentage of biogeografical affinity of fish fauna in the Gulf of California. (PA: Aleutian province, OP: Oregonian province, PS: San Diego province, PC: Cortez province, PM: Mexican province, PP: Panamic province, POT: Oriental tropical pacific, CT: Circuntropical species, T: Transpacific species, END: Endemic species). 
estudio constituye el primer intento por cubrir una porción considerable del fondo demersal en la porción oriental del Golfo de California y sobre todo la composición taxonómica íctica acompañante de la captura de camarón, fauna íctica para la cual no hay ninguna iniciativa de manejo por el momento.

El número de especies registradas es notablemente alto, comparado con la fauna ictiológica demersal de otras áreas del Pacífico mexicano como el Golfo de Tehuantepec (178 especies; Tapia-García \& García Abad 1998), Nayarit, Michoacán, Guerrero (con 174, 120 y 166 especies; Amezcua-Linares 1990) y Jalisco y Colima (161 especies; Mariscal-Romero 2003); sin embargo llama la atención lo reportado por Rodríguez-Romero et al. (2008) de 220 especies en los fondos marinos de la costa occidental de la Península de Baja California, siendo un número elevado en comparación a los señalados y menor al presente estudio. Esta alta riqueza de especies de diferentes requerimientos ecológicos se puede atribuir a los sistemas de corrientes, surgencias, remolinos, topografía y batimetría, que en conjunto originan un ecosistema muy dinámico con un amplio régimen térmico y una gran variedad de hábitat. La amplia cobertura latitudinal y de profundidad abordada en este estudio $(4-137 \mathrm{~m})$ pone de manifiesto que esta región del Golfo de California presenta una gran riqueza de especies de peces, lo que abre diversas expectativas desde el punto de vista ecológico, pesquero, alimenticio y de manejo.

Cabe señalar, que del total de especies registradas (241), tan solo 29 (66\%) fueron frecuentes y abundantes (Cuadro 1), el resto presentaron patrones de cambio estacional o mensual, característica que sobresale en peces tropicales y subtropicales (Rodríguez Romero et al. 1998), gran parte de las especies raras de este estudio corresponden a especies pelágicas y mesopelágicas capturadas de manera accidental durante el lance o levantamiento de la red. Las especies aquí reportadas son características del Pacífico Este (Robertson \& Cramer 2009).
El traslape de distribución geográfica de la fauna íctica es un reflejo de la posición geográfica del Golfo de California, en donde se registran especies de peces tropicales y de zonas templadas a subtropicales, lo que permite el registro de un gran número de especies, tal y como lo han manifestado Galván-Magaña et al. (2000), Mora \& Robertson (2005), Zapata \& Robertson (2007), Rodríguez-Romero et al. (2008), entre otros.

El incremento de especies de peces con registros recientes que se ha presentado en los últimos años, al parecer es una manifestación del cambio climático global y resalta la importancia de explorar nuevas áreas, ayudando a minimizar la incertidumbre del potencial que realmente se tiene en los fondos marinos dentro del ámbito demersal. Las especies del Pacifico Oriental Tropical presentan intrusiones importantes en sus costas, especialmente durante eventos ENSO (El Niño-Oscilación del Sur), que llegan hasta las costas de California cuando son de gran magnitud e intensidad (Pondella 1999, Lea \& Rosenblatt 2000, Moore \& Herbinson 2002 y Walker et al. 2002) como el caso de varias especies con registros recientes en la Provincia de San Diego como el serrano de agua profunda Serranus aequidens, el ronco ofensivo Conodon serrifer y el conejo Caulolatilus affinis, entre otras (Pondella 1999, Moore \& Herbinson 2002, Walker et al. 2002).

Aunque existe un mayor número de especies con afinidad por la región combinada (PS$\mathrm{PP}$ ), se presentaron un total de 18 divisiones zoogeográficas (Fig. 2), que marcan un cambio importante en la asociación, frecuencia y ausencia de las especies de sur a norte (Cuadro 1); lo que indica la amplia variedad de hábitats que presenta el Golfo de California. Al norte sobresalieron especies de afinidad templada, mientras que en el sur predominaron especies típicas del Pacífico Oriental Tropical POT como la vaca angelita (Bellator loxias), el sapo de luto (Porichthys analis), el lenguado zapatilla (Etropus peruvianus), el puerco inicornio Aluterus monoceros, el lenguado leopardo del Pacífico Bothus leopardinus, el serrano mexicano Diplectrum macropoma y otras (Cuadro 
1). Aspectos similares en estas tendencias fueron documentados por Horn et al. (2006) y Rodríguez Romero et al. (2009).

El traslape biogeográfico de la fauna es un reflejo de la posición geográfica del Golfo de California en los límites de distribución de los elementos en donde se manifiestan especies de afinidades templadas, tropicales y de transición templado-cálido, las divisiones que caracterizan al Golfo de California (norte, centro y región sur) y los eventos de corrientes, mareas, turbulencias, surgencias y remolinos que se desarrollan en ella, crean una vigorosa circulación y profundidad variable creando un espectro de energía cinética con periodos bien definidos (López-Martínez et al. 2000), produciendo eventos de alto dinamismo de la fauna y flora marina, donde los peces aprovechan diversos recursos o eventos para establecerse en zonas de alta productividad biológica, desde zonas someras hasta áreas profundas (Rodríguez Romero et al. 1998).

A manera comparativa, se hizo un análisis de las especies de peces dentro de la FAC reportadas por Pérez-Mellado (105 especies) en 1980 con las aquí encontradas, presentándose solo 65 de las 105 especies reportadas ese año, además de otras 175 especies no reportadas. Sin embargo, este resultado no permite llegar a conclusiones sólidas sobre potenciales efectos de la pesca en la biodiversidad, toda vez que en el mencionado trabajo se agruparon especies en géneros (ej. Etropus spp, género en el que se encontró $18 \%$ de los organismos muestreados). Este hecho resalta la importancia de los listados sistemáticos, ya que permiten generar conocimiento sobre la biodiversidad que puede ser comparable en el tiempo con fines de evaluar estados alternos de la comunidad en los ecosistemas.

\section{AGRADECIMIENTOS}

Esta investigación fue financiada por el proyecto SAGARPA-CONACYT 2003-02-089 y el proyecto EP1.1. Se agradece el apoyo de Alejandro Acevedo y de los Laboratorios de Especialidades en Pesquerías y de Ecología de
Peces del CIBNOR y a la Cámara Nacional de la Industria Pesquera de Sonora y la Asociación de Pequeños Armadores de Guaymas por su apoyo para que los observadores a bordo pudieran participar en los viajes de pesca, en especial a la Pesquera Babarasa, Pesquera Delly, Pesquera México, Geomar y Productos Pesqueros de Guaymas.

\section{RESUMEN}

Composición taxonómica de peces integrantes de la fauna de acompañamiento de la pesca industrial de camarón del Golfo de California, México. La pesquería de camarón es la más importante en el Golfo de California y también la más problemática con respecto a las capturas incidentales en México. Dentro de la fauna de acompañamiento del camarón (FAC), el grupo de mayor abundancia son los peces. Se presenta listado sistemático de las especies de peces presentes en la FAC de la pesquería industrial de camarón en la costa oriental del Golfo de California. Durante 2004-2005 se implementó un sistema de observadores a bordo de la flota camaronera de Guaymas, Sonora, que recolectaron muestras de $20 \mathrm{~kg}$ de la captura. Los peces representaron $70 \%$ de las capturas en número. Se recolectó un total de 51101 ejemplares de peces, pertenecientes a dos clases, 20 órdenes, 65 familias, 127 géneros y 241 especies. El orden Perciformes fue el más diverso (31 familias, 78 géneros y 158 especies). Las familias mejor representadas en número de especies fueron: Sciaenidae y Paralichthyidae con 34 y 18 especies, Haemulidae y Carangidae aportaron 16 especies. Los géneros mejor representados fueron Symphurus con nueve y Diplectrum y Cynoscion con seis especies, otros géneros importantes fueron Larimus y Porichthys con cinco especies. El número de especies registrado es alto, comparado con la fauna demersal de otras áreas del Pacífico Mexicano. Esta alta riqueza de especies se puede atribuir a los sistemas de corrientes, surgencias, remolinos, topografía y batimetría únicas, que en conjunto originan un ecosistema dinámico con un amplio régimen térmico y una gran variedad de hábitats. Los resultados aqui presentados ponen de manifiesto que esta región del Golfo de California presenta una gran riqueza de especies, lo que abre diversas expectativas desde el punto de vista ecológico, pesquero, alimenticio y de manejo.

Palabras clave: Golfo de California, ictiofauna, fauna de acompañamiento de camarón.

\section{REFERENCIAS}

Abitía, C.L., R.J. Rodríguez, M.F. Galván, A.J. De la Cruz. \& R.H. Chávez. 1994. Lista sistemática de la 
ictiofauna de Bahía de La Paz, Baja California Sur, México. Cien. Mar. 20: 159-181.

Aguilar-Palomino, B., R.J. Mariscal, S.G. González. \& I.L.E. Rodríguez. 1996. Lista sistemática de la ictiofauna demersal de fondos blandos de la plataforma continental de Jalisco y Colima México, durante mayo y junio de 1995. Cien. Mar. 22: 469-481.

Amezcua-Linares, F. 1990. Los peces demersales de la plataforma continental del Pacífico central de México. Tesis de Doctorado en Ciencias del Mar, Universidad Nacional Autónoma de México, México.

Bojorquez, L.F. 1998. Bycatch utilization in Mexico, p. 21-28. In Report and Proceedings on the FAO/DFID Expert Consultation on Bycatch Utilization in Tropical Fisheries, Bejing, September 1998. Rome, FAO.

Box, E.G., J.H. Stuart \& W.G. Hunter. Estadística para investigadores. Diseño, innovación y descubrimiento. Wiley, Barcelona, España.

Boschi, E.E. 2000. Species of decapod crustaceans and their distribution in the American marine zoogeographic provinces. Rev. Inv. y Des. Pesq. 13: 7-136.

Briggs, J.C. 1974. Marine zoogeography. McGraw-Hill, Nueva York, EEUU.

Briggs, J.C. 1995. Global biogeography. Elsevier, Amsterdam, Holanda.

Castro-Aguirre, J.L., J. Arvizu-Martínez \& J. Páez-Barrera. 1970. Contribución al conocimiento de los peces del Golfo de California. Soc. Mex. de Hist. Nat. 31: 107-181.

Castro-Aguirre, J.L. \& H. Espinosa Pérez. 1996. Listados faunísticos de México. VII. Catálogo sistemático de las rayas y especies afines de México (Chondrichthyes: Elasmobranchii: Rajiformes: Batoideiomorpha). Instituto de Biología, UNAM, D.F., México, México.

Chávez, H. \& J. Arvizu. 1972. Estudio de los recursos pesqueros demersales del Golfo de California, 19681969. III. Fauna de acompañamiento del camarón (peces finos y "basura"), p. 361-378. In Carranza, J. (ed.). Memorias del IV Congreso Nacional de Oceanografía. México, D.F., México.

De La Cruz-Agüero, J., F.M. Galván, A.C. Abitia, J.R. Rodríguez \& F.J. Gutiérrez. 1994. Lista sistemática de los peces marinos de bahía Magdalena, Baja California Sur, México. Cien. Mar. 20: 17-31.

Eschmeyer, W.N., E.S. Herald \& H. Hammann. 1983. A field guide to the Pacific coast fishes of North
America. Hougthon Mifflin, Boston, Massachusetts, EEUU.

Espinosa-Pérez, H., J.L. Castro-Aguirre \& L. Huidobro Campos. 2004. Listados faunísticos de México. IX. Catálogo sistemático de tiburones (Elasmobranchii: Selachimorpha). Instituto de Biología UNAM, D.F., México, México.

Fischer, W., F. Krupp, W. Schneider, C. Sommer, K.E. Carpenter \& V.H. Niem (eds.). 1995. Guía FAO para la identificación de especies para los fines de pesca. Pacifico Centro-Oriental. FAO. Roma. II-III: 648-1652.

Galván-Magaña, F., S.F.J. Gutiérrez, C.L.A. Abitia \& J.R. Rodríguez. 2000. The distribution and affinities of the shore fishes of the Baja California Sur Lagoons, p. 383-398. In M. Munawar, S.G. Laurence, I.F. Munawar \& D.F. Malley (eds.). Aquatic ecosystems of México: status and scoop. Ecovision world monograph. Backhunys Publisher, Leiden, Holanda.

Ginsburg, I. 1958. Flounders of the genus Paralichthys and related genera in American waters. U.S. Fish. Bull. 52: 267-351.

Grainger, R.J.R. \& S.M. Garcia. 1996. Chronicles of marine fishery landings (1950-1994): trend analysis and fisheries potential. FAO Fish Tech. Paper 359: 51.

Horn, M.H., L.G. Allen \& R.N. Lea. 2006. Biogeography, p. 3-25. In L.G. Allen, D.J. Pondella \& M.H. Horn (eds.). The ecology of marine fishes: California and adjacent waters. University of California, Berkeley, California, EEUU.

Hastings, P.A. 2000. Biogeography of the tropical eastern Pacific: distribution and phylogeny of chaenopsid fishes. Zool. J. Lin. Soc. 128: 319-335.

Jordan, D.S. \& B.W. Evermann. 1896-1900. The fishes of North and middle America. Bull. United States Nat. Hist. Mus. 47: 1-3313.

Lea, R.N. \& R.H. Rosenblatt. 2000. Observations on fishes associated with the 1997-98 El Niño off California. CalCOFI Rep. 41: 117-129.

López-Martínez, J. 2000. Dinámica de la pesquería de camarón café (Penaeus californiensis) en el litoral sonorense y su relación con algunos parámetros océano-atmosféricos. Tesis de doctorado, Centro Interdisciplinario de Ciencias Marinas, La Paz, B.C.S., Mexico.

López-Martínez, J., E. Morales-Bojórquez, F. Paredes Mallón, D. Lluch-Belda \& C. Cervantes Valle. 2001. La pesquería de camarón de altamar en Sonora, p. 301-312. In 
D. Lluch-Belda, J. Elorduy-Garay, S. Lluch-Cota \& G. Ponce-Díaz. Centros de Actividad Biológica (BACs) en el Noroeste de México. CIBNOR-CICIMAR-CONACYT, La Paz, B.C.S., México.

Love, M.S., M. Yoklavich \& L. Thorsteinson. 2002. The rockfishes of the northeast Pacific. University of California, Berkeley, EEUU.

Madrid-Vera, J., F. Amezcua \& E. Morales-Bojórquez. 2007. An assessment approach to estimate biomass of fish communities from bycatch data in a tropical shrimp-trawl fishery. Fish. Res. 83: 81-89.

Madrid-Vera, J., A.L. Ruiz \& B.I. Rosado. 1998. Peces de la plataforma continental de Michoacán y sus relaciones en el Pacífico mexicano. Rev. Biol. Trop. 46: 267-276.

Mariscal-Romero, J. 2003. Dinámica de las asociaciones de peces demersales de la plataforma continental de Jalisco y Colima, México. Tesis Doctorado, Universidad de Colima, Colima, México.

McPhail, J.D. 1958. Key to the croakers (Sciaenidae) of the eastern Pacific. Univ. Brit. Columbia, Inst. Fish. Mus. Contrib. 2: 1-20.

Meek, S.E. \& S.F. Hildebrand. 1923-1928. The marine fishes of Panama. Publ. Field. Mus. Nat. Hist., Zool. Ser. 15: 1-1045.

Miller, D.J. \& R.N. Lea. 1976. Guide to the coastal marine fishes of California. Fish. Bull. 157: 1-249.

Moncayo-Estrada, R., J. Castro-Aguirre \& J. De La CruzAgüero. 2006. Lista sistemática de la ictiofauna de Bahía de Banderas, México. Rev. Mex. Biod. 77: 67-80.

Moore, R.H. \& K.T. Herbinson. 2002. First record of the armed grunt, Conodon serrifer (Haemulaide), in southern California. Cal. Fish and Game 88: 178180 .

Mora C. \& D. Robertson. 2005. Causes of latitudinal gradients in species richness: a test with fishes of the Tropical Eastern Pacific. Ecol. 86: 1771-1792.

Nava Romo, J.M. 1995. Impactos a corto, mediano y largo plazo, en la biodiversidad y otras características ecológicas en la comunidad bentónico-demersal capturada por la pesquería del camarón en el norte del Alto Golfo de California, México. Tesis de Maestría, Instituto Tecnológico y de Estudios Superiores de Monterrey, Guaymas, Sonora, México.

Nelson, J.S. 2006. Fishes of the world. Wiley-Hoboken, Nueva Jersey, EEUU.
Nelson, J.S., E.J. Crossman, H. Espinosa-Pérez, L.T. Findley, C.R. Gilbert, R.N. Lea \& J.D. Williams. 2004. Common and scientific names of fishes from the United States, Canada, and Mexico. Amer. Fish. Society, Special Publication 29, Bethesda, Maryland, EEUU.

Orr, J.W., M.A. Brown \& D.C. Baker. 2000. Guide to rockfishes (Scorpaenidae) of the genera Sebastes, Sebastolobus, and Adelosebastes of the Northeast Pacific Ocean. NOAA Tech. Mem.NMFS-AFSC-117: 47.

Pérez Mellado, J. 1980. Análisis de la fauna de acompañamiento del camarón capturado en las costas de Sonora y Sinaloa, México. Tesis de maestría, Instituto Tecnológico y de Estudios Superiores de Monterrey, Escuela de Ciencias Marinas, Guaymas, Sonora, México.

Pérez-Mellado, J., J.M. Romero, R.H. Young \& L.T. Findley. 1982. Yields and composition of by-catch from the Gulf of California, p. 55-57. In FAO/CIID/IDRC (eds.). Fish by-catch-Bonus from the sea. Report of Technical Consultation on shrimp by-catch utilization. Georgetown, Guyana, 27-30 October 1981. Ottawa, Ontario, Canada.

Pondella, D.J. 1999. First record of deepwater bass, Serranus aequidens (Serranidae), from California. Cal. Fish and Game 85: 130-134.

Rábago-Quiroz, C.H., J. López-Martínez, E. Herrera-Valdivia, M.O. Nevarez-Martínez \& J. Rodríguez-Romero. 2008. Population dynamics and spatial distribution of flatfish species in shrimp trawl bycatch in the Gulf of California. Hidrobiol. 18: 193-202.

Robertson, D.R. \& G.R. Allen. 2002. Shore fishes of the Tropical Eastern Pacific: an Information System. Smithsonian Tropical Research Institute, Balboa, Panama.

Robertson, D.R. \& K.L. Cramer. 2009. Shore fishes and biogeographic subdivisions of the Tropical Eastern Pacific. Mar. Ecol. Progr. Ser. 380: 1-17.

Robertson, D.R., J.S. Grove \& J.S. McCosker. 2004. Tropical transpacific shore fishes. Pac. Sci. 58: 507-565.

Rodríguez-Romero J., S. Hernández-Vázquez \& J. LópezMartínez. 2009. Desarrollo potencial de peces desaprovechados. Cien. y Des. 35: 45-51.

Rodríguez-Romero, J., D.S. Palacios-Salgado, J. LópezMartínez, S. Hernández-Vázquez \& G. Ponce-Díaz. 2008. Composición y zoogeografía de los peces demersales de la costa occidental de Baja California Sur, México. Rev. Biol. Trop. 56: 1765-1783. 
Rodríguez-Romero J., A. Abitia-Cárdenas, F. GalvánMagaña, B. Aguilar-Palomino \& J. Arvizu-Martínez. 1998. Ecology of fish community from the soft bottom of Bahia Concepcion, Mexico. Archive of Fishery and Marine Research. Germany 46: 61-76.

Rodríguez-Romero, J., C.L. Abitia, A.J. De la Cruz \& M.F. Gálvan. 1992. Lista sistemática de los peces marinos de Bahía Concepción, Baja California Sur, México. Cien. Mar. 18: 85-95.

Rosales-Casián, J.A. 1996. Ictiofauna de la bahía de San Quintín, Baja California, México, y su costa adyacente. Cien. Mar. 22: 443-458.

Rosenblatt, R.H. \& G.D. Johnson. 1974. Two new species of sea basses of the genus Diplectrum, with a key to the Pacific species. Cal. Fish and Game 60: 178-191.

Tapia-García, M. \& M.C. García-Abad. 1998. Los peces acompañantes del camarón y su potencial como recurso en las costas de Oaxaca y Chiapas, p. 179196. In M. Tapia-García (ed.). El Golfo de Tehuantepec: El ecosistema y sus recursos, UAM, Iztapalapa, D.F., México.
Thomson D.A., L.T. Findley \& A.N. Kerstitch. 1979. Reef fishes of the Sea of Cortez. The Rocky Store fishes of the Gulf of California. Wiley, Nueva York, EEUU.

Van der Heiden A.M. \& L.T. Findley. 1988. Lista de peces marinos del sur de Sinaloa, México. Anales de Cien. del Mar y Limnol. de la Universidad Autónoma de México, México 15: 209-224.

Walker, B.W. 1960. The distribution and affinities of the marine fish fauna of the Gulf of California. Syst. Zool. 9: 123-133.

Walker, H.J. Jr., P.A. Hastings \& R.H. Steele. 2002. The Pacific golden-eyed tilefish, Caulolatilus affinis Gill (Teleostei: Malacanthidae), first occurrence in California. Cal. Fish and Game 88: 139-141.

Walter, H.J. \& R.H. Rosenblatt. 1988. Pacific toadfishes of the genus Porichthys (Batrachoididae) with description of three new species. Copeia 4: 887-904.

Zapata, F.A. \& D.R. Robertson. 2007. How many species of shore fishes are there in the Tropical Eastern Pacific? J. Biog. 34: 38-51. 